\title{
Erratum to: Calculation of the Critical Distances in a System of Two Colliding Nuclei beyond the Monopole Approximation
}

\author{
A. A. Roenko ${ }^{1)^{*}}$ and K. A. Sveshnikov ${ }^{2)}$ \\ Received April 2, 2020; revised April 2, 2020; accepted April 2, 2020
}

DOI: $10.1134 / \mathrm{S} 1063778820300018$

Reference 11 should read as follows:

11. A. Roenko and K. Sveshnikov, Phys. Rev. A 97, 012113 (2018).

https://doi.org/10.1103/PhysRevA.97.012113

The original article can be found online at https://doi.org/10.1134/S1063778819120251

\footnotetext{
1) Bogoliubov Laboratory of Theoretical Physics, Joint Institute for Nuclear Research, Dubna, Moscow oblast, 141980 Russia.

2) Faculty of Physics, Moscow State University, Moscow, 119234 Russia.

*E-mail: roenko@physics.msu.ru
} 